\title{
Seymour fracture: Better do not underestimate it
}

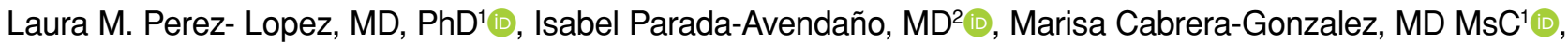 \\ César G. Fontecha, MD PhD'10
}

'Department of Pediatric Orthopaedic Surgery and Traumatology, Sant Joan de Deu Children's Hospital, Barcelona, Spain

${ }^{2}$ Department of Pediatric Orthopaedic Surgery and Traumatology, Miguel Servet University Hospital, Zaragoza, Spain

Seymour fracture was originally described in 1966 as a juxta-epiphyseal distal phalangeal fracture in a child or adolescent, with the dislocated base of the nail plate lying proximally to the nail fold. ${ }^{[1]}$ However, this definition did not reflect the presence or absence of nail bed injury. Rather, the article described it as other "more common open crushing injuries". ${ }^{[1]}$ In the more recent literature, the definition has varied considering Seymour fractures to be inherently open fractures (associated with the avulsion of the nail and a concomitant laceration of the nail bed due to its proximity to the fracture site) ${ }^{[2-4]}$ and distinguishing between open and closed fractures. ${ }^{[5,6]}$ With some contradictions, some sources have identified a similar injury pattern in adults and have included this in their definitions, although this is ambiguous, as adults do not present physis. ${ }^{[5]}$ Radiologically, they can involve the epiphysis (Salter-Harris [SH]

Received: June 30, 2021

Accepted: August 29, 2021

Published online: November 19, 2021

Correspondence: Laura M. Perez-Lopez, MD, PhD. Department of Pediatric Orthopedic Surgery and Traumatology. Sant Joan de Deu Children's Hospital. University of Barcelona, Passeig de Sant Joan de Déu, 2, 08950 Esplugues de Llobregat, Barcelona, Spain.

E-mail: Iperezl@sjdhospitalbarcelona.org

\section{Doi: $10.52312 /$ jdrs.2021.330}

Citation: Perez-Lopez LM, Parada-Avendaño I, Cabrera-Gonzalez M. Fontecha CG. Seymour fracture: Better do not underestimate it. Jt Dis Relat Surg 2021;32(3):569-574.

(2021 All right reserved by the Turkish Joint Diseases Foundation

This is an open access article under the terms of the Creative Commons Attribution-NonCommercial License, which permits use, distribution and reproduction in any medium, provided the original work is properly cited and is not used for commercial purposes (http://creativecommons.org/licenses/by-nc/4.0/).

\section{ABSTRACT}

Objectives: This study aims to analyze the functional results, management, and complications of acute Seymour fracture treatment and to generalize the understanding of Seymour fractures, as well as awareness about its controversial treatment and critical sequelae.

Patients and methods: Between January 1994 and December 2019, a total of 29 patients (20 males, 9 females; mean age: $7.9 \pm 3.9$ years; range, 1 to 15 years) who presented within the first $24 \mathrm{~h}$ of injury and were diagnosed with Seymour fractures and treated in the emergency setting were retrospectively analyzed. Clinical and radiological data were collected from medical records at the time of diagnosis and during follow-up, within a week after the treatment and in the visits required until fracture healing, and no sequelae were observed. In the event of complications, a minimum of one year of follow-up was carried out. Radiographs were taken of the anteroposterior and lateral views during each visit.

Results: The mean follow-up was 10.8 \pm 8.6 (range, 2 to 36) months. Surgical treatment in the operating room was performed in $24(82.7 \%)$ patients using a single longitudinal Kirschner wire (K-wire) fixation through the distal phalanx and the distal interphalangeal joint in 21 patients. Non-operative treatment based on closed reduction and splinting was performed in five $(17.3 \%)$ patients. There was no statistically significant difference in the final passive range of motion and physeal growth arrest in relation to the use or non-use of K-wires. The use of antibiotics in any of the three possible administrations (intravenous antibiotic regimen, intravenous and later oral antibiotic at-home or oral antibiotics), in relation to the non-use of antibiotics seemed to be a protective factor against infections (odds ratio $=0.04 ; 95 \%$ confidence interval: $0.006-0.2 ; \mathrm{p}=0.001)$.

Conclusion: The identification of Seymour fractures is crucial for applying the correct treatment and reducing the risk of complications, such as osteomyelitis and physeal alterations. Based on our study results, we can suggest that the use of an antibiotic regimen causes a lower risk of infections in acute Seymour fractures. The prompt identification of these fractures with a standardized protocol covering irrigation, debridement, reduction, fixation, and prophylactic antibiotics is needed to avoid complications.

Keywords: Distal phalanx, infection, Juxta-epiphyseal fracture, osteomyelitis, phalanx fracture, Seymour fracture. 
types I and II) or the metaphysis just 1 to $2 \mathrm{~mm}$ distal to the physis (juxta-epiphyseal).

Owing to the asymmetry of tendon insertions where the extensor pollicis longus is inserted into the epiphysis, and where the flexor digitorum profundus is inserted into the metaphysis, this injury seems to be a pseudo-mallet with the flexion deformity of the distal phalanx. However, it does not involve a tear or avulsion of the extensor tendon. ${ }^{[6]}$ A lateral $\mathrm{X}$-ray is helpful to differentiate it from Seymour fractures. The incidence of Seymour fractures has never been reported; only Rask et al. ${ }^{[7]}$ estimated its prevalence as $5.4 \%$ among distal phalanx fractures at a local institution over a 15 -year period. The most common mechanisms of injury are the crushing or entrapment of the digit in a closing door or swing, or sport-related injuries, ${ }^{[2,5-9]}$ including the newly emerging recreational/transportation vehicle, the hoverboard ${ }^{[4]}$ among other causes. As in the rest of phalanx fractures in children, the middle finger is the most commonly involved. ${ }^{[10]}$

Given the variety of presentations, from a subtle nailbed injury with minimally displaced features to a grossly displaced and exposed fracture, it can be difficult to notice the severity of these injuries. Currently, no consensus exists regarding the optimal treatment. The management options range from closed reductions and splinting to following the principles of an open fracture including irrigation, surgical debridement, fracture reduction, nail bed repair, and antibiotic administration. The latter has become a more widely accepted practice in more recent years, but without a specific protocol. . $^{[2,5,6,11]}$ Following our experience and what the literature reports, the failure to recognize Seymour fractures may lead to complications such as osteomyelitis, physeal growth alterations, and/or nail aesthetic sequelae/dystrophy. ${ }^{[12]}$

In the present study, we aimed to analyze the functional results, management, and complications of acute Seymour fracture treatment and to generalize the understanding of Seymour fractures, as well as awareness about its controversial treatment and critical sequelae.

\section{PATIENTS AND METHODS}

This single-center, retrospective, observational study was conducted at Sant Joan de Deu Children's Hospital, Department of Paediatric Orthopaedic Surgery and Traumatology between January 1994 and December 2019. Inclusion criteria were as follows: patients younger than 18 years old, having a diagnosis and receiving treatment in the emergency setting for acute Seymour fractures, presenting within the first $24 \mathrm{~h}$ of injury, with or without associated lesions. A Seymour fracture was diagnosed radiographically as evidence of the juxta-epiphyseal or the Salter-Harris I or II fracture of the distal phalanx clinically associated with an open nailbed injury. Patients with previous injuries to the same epiphyseal plate or comminuted distal bony fragments and those who were lost to follow-up were excluded. Finally, a total of 29 patients ( 20 males, 9 females; mean age: $7.9 \pm 3.9$ years; range, 1 to 15 years) were included. A written informed consent was obtained from each parent and/or legal guardian of the patient. The study protocol was approved by the Sant Joan de Deu Children's Hospital Ethics Committee (No: EPA 22-20). The study was conducted in accordance with the principles of the Declaration of Helsinki.

Clinical and radiological data were collected from medical records at the time of diagnosis and during follow-up, within a week after the treatment and in the visits required until fracture healing, and no sequelae were observed. In the event of complications, a minimum of one year of follow-up was carried out. Radiographs were taken of the anteroposterior and lateral views during each visit.

Data including sex and age at the time of diagnosis, the affected finger and hand side, the cause of the fracture, the type of fracture in the X-ray, treatment management, fixation with Kirschner wire (K-wire), antibiotic management, the functional result, and complications were recorded. Currently, the protocol for the management of Seymour fractures is not clear in the literature and, therefore, heterogeneity existed in our clinical practice data regarding surgical steps, K-wire fixation, and antibiotic regimen. We divided these patients into two groups: those receiving nonoperative treatment in the emergency room using local anesthesia via a digital block with/without conscious sedation, closed reduction, and splints $(n=5)$ and patients with surgical treatment in the operating room $(n=24)$. Regarding antibiotic management, adjusted based on the patient's weight, we distinguished among an intravenous antibiotic regimen (a single dose of amoxicillinclavulanate), an intravenous antibiotic regimen and a later oral antibiotic at home (an initial intravenous dose of amoxicillin-clavulanate in the emergency room and then every $8 \mathrm{~h}$ during a 24 -h period followed by an oral administration with the same 
interval across $72 \mathrm{~h}$ at home), oral antibiotics only (three days of amoxicillin-clavulanate every $8 \mathrm{~h}$ ), and no antibiotics. The functional result, clinically assessed with the range of motion (ROM) of the distal interphalangeal joint (DIPJ), was compared with the ROM of the contralateral DIPJ finger with the results of normal or altered. Complications that were taken into account in data collection were superficial infection determined by such clinical signs as pain, swelling, warmth, and erythema; deep infection based on gross purulence; and osteomyelitis described as infection signs associated with radiographs' bony erosion, a periosteal reaction, and sclerotic bone, as well as nuclear magnetic resonance (NMR) diagnosis. In cases with a minimum follow-up of nine months, nonunion was evaluated along with nail or physeal growth alterations with a minimum follow-up of three months after the injury. Secondary displacement and the time of fracture healing were also analyzed. The use of K-wires in relation to the final ROM and growth arrest was also examined.

\section{Statistical analysis}

Statistical analysis was performed using the IBM SPSS version 23.0 software (IBM Corp., Armonk, NY, USA). Descriptive data were expressed in mean \pm standard deviation (SD) or median (min-max) for continuous variables and in number and frequency for categorical variables. The Fisher exact test was used to compare the categorical variables. A $p$ value of $<0.05$ was considered statistically significant with odds ratio (OR) and 95\% confidence interval (CI).

\section{RESULTS}

The mean follow-up was $10.8 \pm 8.6$ (range, 2 to 36 ) months. The injury affected the left hand in $68 \%$ of cases $(n=20)$, with the middle finger being the most commonly involved in $31 \%$ of cases $(n=9)$, followed by the thumb and the index finger with $20.6 \%$ of cases $(n=6)$ for each one and $27.5 \%$ for the small fingers $(n=8)$. Entrapment of the digit in a closing door and contusions were the mechanisms in these lesions. Radiologically, the fracture type was distributed as $65.5 \%(\mathrm{n}=19)$ for the $\mathrm{SH}$ type II (Figure $1 \mathrm{a}, \mathrm{b})$ and $34.5 \%(\mathrm{n}=10)$ for the SH type I. Ten patients presented associated lesions as the complete nail avulsion $(n=6)$ (Figure 2), deep flexor tendon lesion in zone $1(\mathrm{n}=1)$, fractures and wounds in other digits $(n=2)$, and with neurovascular lesions $(n=1)$.

Surgical treatment in the operating room was performed in $24(82.7 \%)$ patients with common steps, such as debridement, open reduction, nail bed suture (which was mostly required), and nail plate

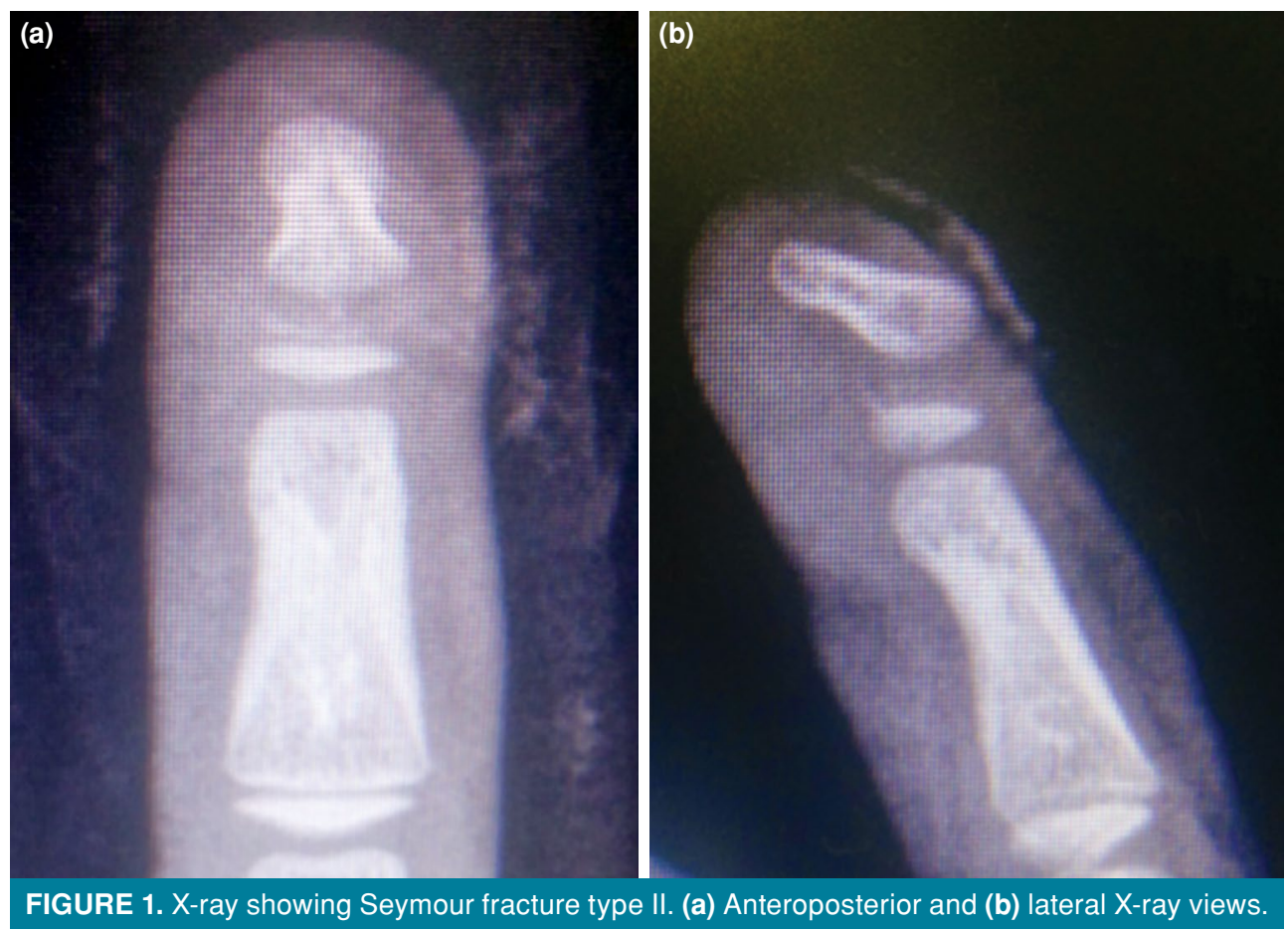



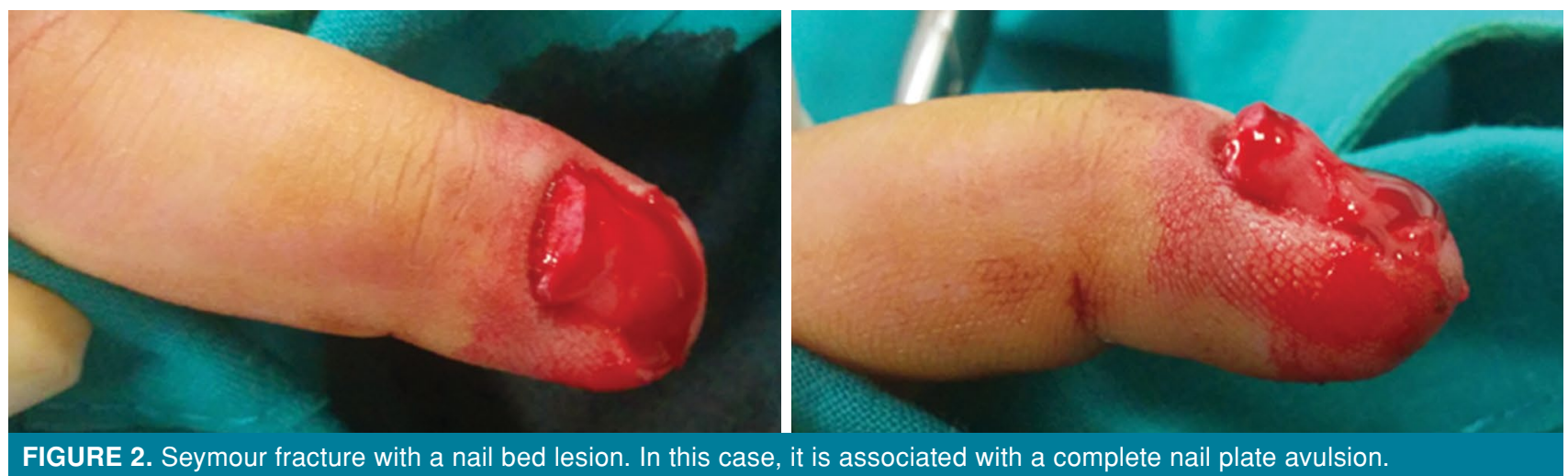

FIGURE 2. Seymour fracture with a nail bed lesion. In this case, it is associated with a complete nail plate avulsion.

fixation. Fracture fixation with a single longitudinal K-wire through the distal phalanx and the DIPJ was performed in $21 / 24(87.5 \%)$ patients. For the remaining three cases, the reduction was maintained with a splint or cast. Non-operative treatment based on closed reduction and splinting was performed in five (17.3\%) patients.

The various forms of antibiotic management were divided into four groups: (i) early intravenous antibiotic regimen before $12 \mathrm{~h}$ after a lesion, was administered in $41.3 \%(n=12)$ of the patients, where one case of osteomyelitis, two cases of abnormal final ROM, and six cases of the physeal growth arrest of the distal phalanx were found during follow-up; (ii) early intravenous and later oral antibiotics prescribed at home were administered in $41.3 \%(n=12)$ with two cases of physeal growth arrest; (iii) oral antibiotic was followed by only one (3.4\%) patient, in which lysis around the K-wire was noticed in the X-ray, but with no other complications added; (iv) no antibiotic regimen group represented $13.7 \%$ of the patients $(n=4)$, with complications in all of them described as two cases of osteomyelitis and two others with osteomyelitis, associated with premature physeal closure and abnormal final $\mathrm{ROM}$ in one case and interphalangeal arthrodesis in the other. Among these four patients, three were treated non-operatively with closed reduction and splinting without using fixation with a K-wire. Regarding the use of a K-wire in our study, $72.4 \%$ $(n=21)$ of our patient cohort had the fixation of the fracture with this method, and one (4.7\%) patient who was not prescribed antibiotics had an infection. In the remaining eight (27.6\%) patients, where the K-wire was not used, we described four (50\%) infections, three of them without a prescribed antibiotic regimen.
We statistically compared the use or non-use of K-wire in relation to a normal final ROM and growth arrest, respectively. The results showed that the percentage of cases with normal final passive ROM did not have significant differences, either, whether a K-wire was present $(90.5 \%)$ or not $(75 \%)$ (OR: 3.1; 95\% CI: 0.36-27.5; $p=0.3$ ). Although physeal growth arrest seems to be more frequent in cases with K-wire $(42.9 \%)$ than without K-wire $(12.5 \%)$, no statistically significant difference was observed (OR: 5.25; $95 \%$ CI: 0.5-50.6; $\mathrm{p}=0.201$ ).

Suffering from infectious osteomyelitis seems to be less probable when a K-wire is used as a synthesis method (4.8\%), rather than when not (50\%) $(\mathrm{p}=0.013$; OR: 0.050). However, before considering K-wire as a protective factor, we must take into account biases such as the non-inclusion in the comparative analysis of more possible factors (antibiotics, debridement, nailbed suture) due to the design of study and the small sample size.

Regarding types of antibiotic management concerning the same variables as before, the results showed no significant relationship between the different antibiotic regimens and the final passive $\mathrm{ROM}(\mathrm{p}>0.05)$. Similar results were found with regard to physeal growth arrest $(p>0.05)$. There was no significant relationship, either, between infection rates and early intravenous antibiotic management, not even when it was compared with the oral antibiotic association, although this combination had an infection rate of $0 \%$ in comparison with the rest of the treatments (29.4\%) (OR: 1.4; 95\% CI: 0.02-3.04; $\mathrm{p}=0.05$ ). Finally, we observed the fact that the use of antibiotics in any of the three possible administrations, in relation to the non-use of antibiotics, led us to the conclusion that their use could be deemed a protective 
factor against infections (OR: 0.04; 95\% CI: 0.006-0.2; $\mathrm{p}=0.001$ ).

\section{DISCUSSION}

Currently, a range of management options for acute Seymour fractures have been reported in the literature, from closed reduction and splinting to operative treatment. No significant difference in the rate of infections has been reported when stable reduction is achieved, under conscious sedation and local anesthesia in the emergency department, ${ }^{[3,7,9]}$ or under general anesthesia in an operating room. ${ }^{[2,11]}$

A large variety of steps (in relation to nail plate removal, eponychial fold, K-wire fixation, and splinting or casting techniques) have been described in operative treatment, although most authors attempt to follow the principles of open fractures. ${ }^{[2-4,7,9]}$ It starts with intravenous antibiotic coverage (cephalosporin). Some authors remove the nail plate carefully for a more extensive irrigation, debridement, and better nailbed repair, replacing it again at the end. ${ }^{[9]}$ However, others avoid removing it and make several round holes on it to drain the hematoma without repairing the nail bed. ${ }^{[13]}$ Often irrigation and debridement are not done, either. Before reduction, any interposed soft tissue should be removed from the fracture line with maneuvers such as the hyperflexion of the digit or distal fragment retraction with a skin hook ${ }^{[9,13]}$ and it is recommended to make a flap through two incisions perpendicular to the eponychium, if any obstacle exists. $^{[6]}$ Nail bed laceration can be repaired with 6-0 or 7-0 absorbable sutures. ${ }^{[1]}$ Applying a force in hyperextension allows one to replace the nail under the proximal nail fold and to suture it. It obtains a stable reduction in most of the cases by acting like a splint.

The K-wire fixation through the distal phalanx and the DIPJ is also controversial. Some authors, as Seymour, consider it a cause of complications (infections, nail deformities, and growth distal phalanx arrest) and utilize the simple suturing of the nail plate and a splint for stabilization, ${ }^{[1,7,13]}$ while some others suggest that early irrigation, debridement, and the administration of antibiotics can prevent them and K-wire fixation is a good option, if the fracture is unstable or if the patient has a possible poor compliance. ${ }^{[3,5,14]}$ KruscheMandl et al. ${ }^{[2]}$ reported that the premature closure of the physis was usually secondary to infection rather than to the direct injury of the growth plate by K-wires. Although our study has not reached statistically significant differences with respect to the final passive ROM or physeal growth arrest, the percentages with the use of K-wire seem to be better in the final passive ROM, but with a higher frequency of physeal growth arrest.

In our study, suffering from infectious osteomyelitis seems to be less probable when a $\mathrm{K}$-wire is used as a synthesis method, rather than when not ( $\mathrm{p}=0.013$, OR: 0.050).

There is also no consensus on the antibiotic regimen after surgery in cases where it is prescribed, being described from three days intravenous antibiotic administration (cephalosporines) ${ }^{[6,12]}$ to oral antibiotics upon discharge during four to 10 days. ${ }^{[2,3,9]}$ The most common oral antibiotics prescribed for an acute Seymour fracture are cephalexin, cefazolin, clindamycin, trimethroprim/sulfamethoxazole, and amoxicillin-clavulanate, among others. ${ }^{[7]}$ The latter is the one used preferably in our center following the same guideline as for open fractures treatment. The preferred antibiotic for delayed Seymour fracture treatment, described in the literature, is clindamycin instead of cephalexin, likely due to the improved osseous penetration improving its effectiveness against osteomyelitis and facilitating fracture healing. ${ }^{[8]}$

Patients who underwent prompt antibiotic administration (within $24 \mathrm{~h}$ after injury), irrigation, debridement and fracture reduction can significantly reduce the rates of infections. ${ }^{\left[{ }^{7,9]}\right.}$ It seems to be that antibiotic administration is protective against nonunion or delayed union $^{[8]}$ and infection, as in our study.

Non-union can also be prevented, withdrawing any interposed nail bed from the fracture site. Concerning nail deformities, it has been reported that they are caused by injury to the nail bed rather than by different treatment methods, ${ }^{[2]}$ although many studies have an insufficient follow-up to assess this complication.

The lack of a standardized treatment protocol, antibiotic administration, and clinical assessment at follow-up, in our review and most of the articles published, implies a high variable of treatment regimens that do not allow us to analyze significantly the efficacy, risks, and complications of all of them. Besides, the severity of the associated injuries may have influenced the results and it is not sufficiently documented in the literature. Finally, small samples size in this study led to the inadequate power to perform statistical comparisons between groups or variables. Therefore, future prospective studies using common criteria should be performed to confirm these data. 
In conclusion, the identification of Seymour fractures is crucial to apply the correct treatment, decreasing the risk of complications, such as osteomyelitis or physeal alterations. According to our results, a lower risk of infections in acute Seymour fractures is achieved with the use of antibiotics, with the intravenous and later oral antibiotic regimen being the preferred option. The prompt identification of these fractures with a standardized protocol covering irrigation, debridement, reduction, and prophylactic antibiotics is needed to avoid complications.

\section{Declaration of conflicting interests}

The authors declared no conflicts of interest with respect to the authorship and/or publication of this article.

\section{Funding}

The authors received no financial support for the research and/or authorship of this article.

\section{REFERENCES}

1. Seymour N. Juxta-epiphysial fracture of the terminal phalanx of the finger. J Bone Joint Surg [Br] 1966;48:347-9.

2. Krusche-Mandl I, Köttstorfer J, Thalhammer G, Aldrian S, Erhart J, Platzer P. Seymour fractures: Retrospective analysis and therapeutic considerations. J Hand Surg Am 2013;38:258-64.

3. Lin JS, Popp JE, Balch Samora J. Treatment of acute Seymour fractures. J Pediatr Orthop 2019;39:e23-e27.

4. Kattan AE, AlShomer F, Alhujayri AK, Alfowzan M, Murrad KA, Alsajjan H. A case series of pediatric seymour fractures related to hoverboards: Increasing trend with changing lifestyle. Int J Surg Case Rep 2017;38:57-60.

5. Al-Qattan MM. Extra-articular transverse fractures of the base of the distal phalanx (Seymour's fracture) in children and adults. J Hand Surg Br 2001;26:201-6.

6. Ganayem M, Edelson G. Base of distal phalanx fracture in children: A mallet finger mimic. J Pediatr Orthop 2005;25:487-9.

7. Rask DMG, Wingfield J, Elrick B, Chen C, Lalka A, Sibbel SE, et al. Seymour fractures: A retrospective review of infection rates, treatment and timing of antibiotic administration. Pediatr Emerg Care 2020.

8. Samade R, Lin JS, Popp JE, Samora JB. Delayed presentation of Seymour fractures: A single institution experience and management recommendations. Hand (N Y) 2021;16:686-93.

9. Reyes BA, Ho CA. The high risk of infection with delayed treatment of open Seymour fractures: Salter-Harris I/II or juxta-epiphyseal fractures of the distal phalanx with associated nailbed laceration. J Pediatr Orthop 2017;37:247-53.

10. Nellans KW, Chung KC. Pediatric hand fractures. Hand Clin 2013;29:569-78.

11. Abzug JM, Kozin SH. Seymour fractures. J Hand Surg Am 2013;38:2267-70.

12. Atik OŞ. Is there something new and interesting in my article? Eklem Hastalik Cerrahisi 2019;30:69.

13. Cha SM, Kang JW, Shin HD, Ga IH. Acute Seymour fractures in children/adolescents overlooked as a simple nail injury: Reduction without pinning. Hand Surg Rehabil 2021;40:171-6.

14. Zhang L, Zuo Y, Wang Y, Zhou H, Wang G, Yu T, et al. Surgical treatment of Seymour fractures in children and adolescents. Zhongguo Xiu Fu Chong Jian Wai Ke Za Zhi 2016;30:529-31. 